\title{
Towards a resilient health status assessment employing intelligence in cyber physical systems
}

\author{
${\text { Maria } \text { Krizea }^{l}, \text { John Gialelis }}^{1,2}$, Christos Panagiotou ${ }^{1}$, and Stavros Koubias ${ }^{l}$ \\ ${ }^{1}$ University of Patras, Electrical and Computer Engineering Department, Applied Electronics \\ Laboratory, University Campus, Rio Patras, Greece \\ ${ }^{2}$ Industrial Systems Institute/ATHENA RC, Platani, Patras, Greece \\ mariakrizea@gmail.com, gialelis@ece.upatras.gr, chpanag@gmail.com, koubias@ece.upatras.gr
}

\begin{abstract}
Resilience is defined as the capacity of a system to cope with a hazardous event or disturbance, responding or reorganizing in ways that maintain its essential function, identity, and structure, while also maintaining the capacity for adaptation, learning and transformation. A resilient health system is one that is capable to anticipate, respond to, cope with, recover from and adapt to system-related shocks and stress, so as to bring sustained improvements in population health, despite the unstable circumstances. Nowadays, the emergency department (ED) of hospitals faces growing demands, rising acuteness and longer waiting times. An efficient, accurate and resilient triage system to improve the operation of the ED becomes crucial. In this work, a resilient system for automatic priority, sorting and monitoring of medical events -triage system- is developed, so that the patients in the ED are treated according to the severity of their condition and not by the order of attendance utilizing a Fuzzy Inference System (FIS) that aggregates, processes patients' vital signs as well as determines their Health Status (HS), according to which the ED staff performs the appropriate classification.
\end{abstract}

\section{Introduction}

The ED is an autonomous part of the hospital that aims to receive, revitalize, diagnose and treat patients with a variety of emergencies that cover the full range of diseases, injuries and disorders. Effective emergency response is a matter of utmost importance for the reliability of every health system. Nowadays, the ED faces serious problems due to the massive occurrence of incidents that create crowded conditions, increased waiting times and other undesirable effects. Increasing needs and requirements and increasing demands for healthcare monitoring and patient assistance pose challenges that need to be addressed by improving current health systems and infrastructures. The use of technology contributes effective and decisive to enhance the operation of ED. The need to provide a high level of medical services and the need for rapid and reliable recording of medical and other data, which data in ED can even judge the outcome of an incident, make the use of an appropriately adapted information system imperative. The development and exploitation of such a system is a significant improvement in the operation of the ED. During ED crowding and all the malfunctions it causes there is an imbalance between the need for emergency care and available resources, which enhances the need for accurate triage [1]. Triage is a process that is critical to the effective management of modern EDs. Triage systems are used in EDs to prioritize patients and to ensure that they are seen in order of clinical need when demand exceeds capacity. They do this by determining who will not be disadvantaged by 
longer waiting times and who requires immediate attention and urgent medical care to achieve optimal outcomes. There are several protocols and methods that are used for triage in EDs (ED Triage), which differ in both patient assessment criteria and the number of categorization levels. Thus, there are ED Triage systems with two, three, four or five level scale. Collective experience and research has reinforced the value of a five level categorical scale [2]. Triage systems aim, not only to ensure clinical justice for the patient, but also to provide an effective tool for departmental organisation, monitoring and evaluation.

The earliest written record of the use of triage in emergency medicine, in a systematic sense, was in the early 1960s at Baltimore, USA [3]. However, this and other early systems lacked formal structure and organisation. In addition, there was no agreement on the categories used. Over time, many departments began to introduce more formalised systems of Triage to meet the demands of modern emergency medicine. Over the last 30 years, triage systems have been standardised in a number of countries and efforts made to ensure consistency of application [4]. A triage scale, which incorporated a patient "urgency test" to discriminate between triage categories, was introduced into the ED at Ipswich Hospital, Queensland, Australia. The Ipswich Triage Scale (ITS) was a five category scale similar to one in use at the Box Hill Hospital, Victoria, Australia. Validation of the ITS led to its adoption as the National Triage Scale (NTS) [5-6] and subsequently the Australasian Triage Scale (ATS) [6-7]. This in turn formed the basis of the Manchester Triage Scale (MTS) [8] in the UK and the Canadian Triage and Acuity Scale (CTAS) [9-11] in Canada. Several international scales have developed from or been based upon the ATS. Most EDs in the USA continue to use a three-level scale (emergency, urgent and non-urgent) but, increasingly, five level scales are being adopted throughout the country [12]. The most common is the Emergency Severity Index (ESI) which is also a five-level categorical scale. Other countries, or at least individual institutions, have adopted these scales including the CTAS in Sweden [13], Andorra [14] and the Netherlands [15] and the ESI in Greece [16].

In this study, a resilient triage system that can be used for priority, sorting and tracking medical incidents is developed allowing classification and prioritization of patients based on the severity of their condition. The application aggregates and processes specific vital signs (HR, RR, BSP) from patients arriving at ED and generates results about their HS, effectively contributing to the identification of emergencies and their classification, with regard to the priority, by the appropriate staff of the ED. Through the system, automated, efficient, reliable and low-cost sorting and monitoring of medical incidents is achieved.

\section{System Description}

With purpose to enhancing the operation of EDs an artificial intelligence system based on Fuzzy Logic (FL) is developed. The system uses and processes vital signs of patients / samples, which is pumped from Physio Net's Massachusetts General Hospital / Marquette Foundation (MGH / MF) Waveform Database [17]. The information that are used by the Database are electrocardiogram (ECG), RR (breaths per minute), BSP (mmHg) and AGE for each patient. The HR (beats per minute) is calculated by processing the ECG through the application. The vital signs are given as input parameters to a FIS [18-19], which determines the patient's HS, based on scientifically and medically documented rules implemented by the application algorithm and according to the fuzzy control system architecture [20-21].

The ECG is the display of the electrical activity of the heart. Basic shape of ECG signal is shown in Figure 1. The recorded ECG signal is often affected by ambient noise (powerline, electrode noise, respiration rate, cardiac muscle movement). Isoelectric line of ECG signal 
is often affected by the artifacts from breathing or electrode phenomena. Moreover, the ECG signal presents powerline noise $(50 \mathrm{~Hz} / 60 \mathrm{~Hz})$. In accordance with the purpose and needs of each application, signal processing involves the usage of different types of filter [22-23]. Filter evaluations is accomplished with regard to achievement of distortion filtration and with regard to minimal additional deformation of ECG signal shape. Fluctuations of isoelectric line can be suppressed using high-pass (HP) filters. The critical point of filter design is the choice of filter band-pass, since the low frequency components of ECG signal are important to keep the correct shape of ST segment. According to the American Heart Association, the demand on the analog ECG signal recording is frequency band from $0.05 \mathrm{~Hz}$ to $100 \mathrm{~Hz}$, however this range is doubtful [24]. According to the other articles, even cut-off frequency of $2 \mathrm{~Hz}$ does not cause visible changes of ST segment [25]. The filter that applied to this algorithm is an IIR Butterworths band-pass filter (second order). This band-pass filter is designed to fade all frequencies below $2 \mathrm{~Hz}$ and beyond 40 $\mathrm{Hz}$. In this way, most of the noise recorded is weakened.

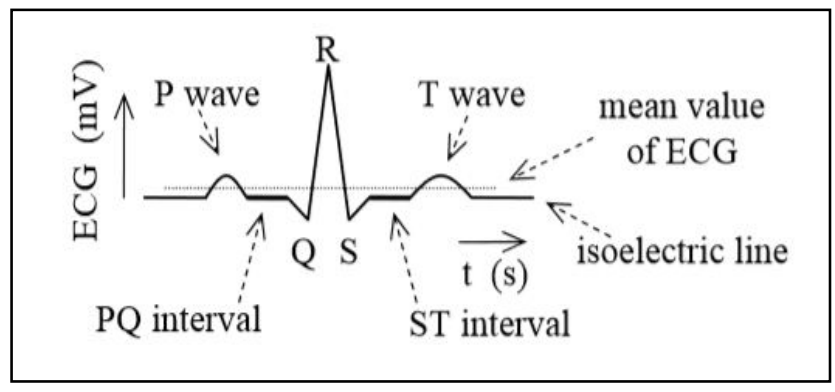

Figure 1. Schematic illustration of ECG signal components

The application applies the Butterworths band-pass filter to the ECG signal that derived from the Physionet Database and subsequently determines the HR through a specific algorithm. The HR along with the other data received from the Database (RR, BSP, AGE) are given as input parameters to a FIS, which executes its rules for the given inputs in each case of patient and determines the output for the patient HS. HS receives three values critical, bad, good. Critical HS declares that the patient needs immediate attention and urgent medical care, bad HS states that the patient need medical treatment but not urgent, so can wait for a short time and good HS means that the patient can wait for his treatment about a longer time without jeopardizing his health. According to the categorization of incidents through the HS the appropriate staff of the ED identifies the emergencies and classifies the patients depending on their need of medical care.

Two FIS are developed. The inputs of the first FIS are HR, RR, BSP and AGE, while the inputs of the second system are the above mentioned inputs, excluding BSP. Each system implements different rules according to its input parameters. The rules are synthesized based on the knowledge that exists for human vital signs and their age-appropriate physiological scales, as shown in Figure 2. Knowledge derives from in-depth research and studies and from medical and scientific evidence [26]. The 4-ipnuts FIS implements 72 rules, while the 3 -inputs FIS implements 36 rules. These rules combine the vital signs with the corresponding values they can obtain according to age and through their execution the patient's HS is determined. A sample of 3-inputs FIS rules is given below.

IF (AGE) IS adult AND (RR) IS fast AND (HR) IS high THEN (HS) IS critical

IF (AGE) IS adult AND (RR) IS average AND (HR) IS low THEN (HS) IS bad

IF (AGE) IS adult AND (RR) IS slow AND (HR) IS low THEN (HS) IS critical IF (AGE) IS pre-schooler AND (RR) IS fast AND (HR) IS normal THEN (HS) IS bad 
IF (AGE) IS pre-schooler AND (RR) IS fast AND (HR) IS high THEN (HS) IS good

IF (AGE) IS school age AND (RR) IS slow AND (HR) IS normal THEN (HS) IS bad

IF (AGE) IS adolescent AND (RR) IS average AND (HR) IS normal THEN (HS) IS good

IF (AGE) IS infant AND (RR) IS fast AND (HR) IS low THEN (HS) IS critical

\begin{tabular}{|c|c|c|c|c|}
\hline Approximate Age Range & Heart Rate & Respiratory Rate & \multirow[b]{3}{*}{ Approximate Age Range } & \multirow[b]{3}{*}{ Systolic Range } \\
\hline Newborn & $100-160$ & $30-50$ & & \\
\hline $0-5$ months & & & & \\
\hline & & & 1-12 months & $75-100$ \\
\hline 6-12 months & $80-140$ & $20=30$ & \multirow{2}{*}{$1-4$ years } & \multirow{2}{*}{$80-110$} \\
\hline $1-3$ years & 80-130 & $20-30$ & & \\
\hline $3-5$ years & $80-120$ & $20-30$ & 3-5 years & 80-110 \\
\hline $6-10$ years & $70-110$ & $15-30$ & $6-13$ years & $85-120$ \\
\hline & & & \multirow{2}{*}{$13-18$ years } & $95-140$ \\
\hline Hilt years & 60-105 & $12-20$ & & hadults is 120 (systolic) \\
\hline $15-20$ years & $60-100$ & $12-30$ & & \\
\hline Adults & $50-80$ & $16-20$ & & \\
\hline
\end{tabular}

Figure 2. Normal vital ranges according to age

\section{System Functionality}

A proper designed and useful triage system focus on automate information and classify patients based on the severity of their condition. The ultimate goal of such a system is to simulate human logic and facilitate the role of staff of EDs. In our case, variables such as vital signs are by definition fuzzy concepts and as a consequence a FIS can describe them more effectively. Moreover, the physiology of patients can be affected by factors such as gender, emotional and physical condition, et al. Thus, experts need a precise tool that examines these factors giving a reliable result. As it seems, FL can satisfy the intent of this application. The features of FIS meet the needs of the application and its usage is deemed suitable.

The training of the system is made possible through a special purpose application that was built aiming at using and processing as limited inputs / data as possible but achieving a correct and valid result. In our case this limitation is realistic, since in emergency rooms some of the vital signs are difficult to collect (e.g., BSP). Thus, the system is functional and, considering the reliability of the output it provides, can become a useful health status control system. The reliability of the system is approved by the control of its results. Moreover, the system is designed to be resilient as it provides equally reliable results for health status with the lack of an information parameter, which in our case is the BSP. With pursue of reducing input data as well as considering the resilience of system, two FIS are developed with one of them having an input less (BSP is excluded). The results of each of two FIS were compared with the expected results which are available from the MGH / MF Waveform Database. The comparison proved the validity of both FIS as they provide reliable information for the patient's health status. Accordingly, the use of both systems is considered beneficial and suitable for the purposes of the application. However, the results of two FIS were compared each other. The FIS present a $18 \%$ variation in the results they provide. The divergence between the outcomes of two FIS for the patients/samples that are used is presented in Figure 3. 


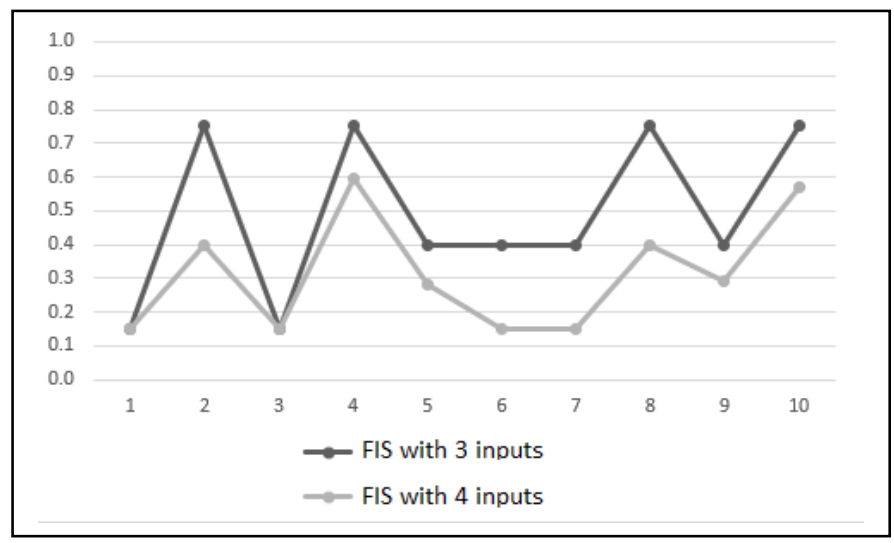

Figure 3. FIS variation

By checking the results and comparing them, we notice that the differences between them are acceptable, as the divergence is small and not able to distort the result that is expected in each case and lead to erroneous conclusions. Therefore, the divergence is satisfactory as the FIS with reduced inputs offers valid conclusions about the patients HS, providing a control system that is simple to implement, flexible and reliable at the same time.

\section{Discussion}

In essence, two applications are being developed. The 4-inputs FIS is used for the training of the system and the 3-inputs FIS is used for the assessment of patient's health. Training is achieved through the evaluation of the results of the first application compared with the expected results which are available from the MGH / MF Waveform Database. The evaluation demonstrated the reliability of 4-inputs FIS for the outcomes of patients HS it provides. Considering the resilience, we exclude the BSP as input, since in emergency rooms BSP is difficult to collect, and we developed the 3-ipnuts FIS. The second application with limited inputs / data provides results with a $18 \%$ variation from the first. This variation is acceptable as the evaluation of the results of the second FIS proved that they remain reliable. More specifically, due to the lack of an information parameter the result is different in some cases. HS outcomes of the two FIS are shown in Figure 4. Observing the results, it appears that this difference does not lead to incorrect conclusions.

\begin{tabular}{|l|c|c|}
\hline & FIS with 4 inputs & FIS with 3 inputs \\
\hline Patient/Sample 1 & critical & critical \\
\hline Patient/Sample 2 & bad & good \\
\hline Patient/Sample 3 & critical & critical \\
\hline Patient/Sample 4 & bad to good & good \\
\hline Patient/Sample 5 & critical to bad & bad \\
\hline Patient/Sample 6 & critical & bad \\
\hline Patient/Sample 7 & critical & good \\
\hline Patient/Sample 8 & bad & bad \\
\hline Patient/Sample 9 & critical to bad & good \\
\hline Patient/Sample 10 & bad to good & \\
\hline
\end{tabular}

Figure 4. FIS results for patients Health Status

The application is a robust tool that can be used for medical purposes. Beyond the specific function it performs for the present work, it has features that favor its use as a basis for 
further improvement and development. The application is open source, its software is flexible, allows for easy and fast code integration and offers the ability to easily transfer and support different systems and devices. Also, the application is expandable and can be reused by incorporating new features and implementing new processes.

\section{Conclusions}

Triage has become an integral part of the function of EDs around the world and has demonstrated clinical and organisational value. This paper describes a resilient triage system that can be used for priority, sorting and tracking medical incidents developed to allow classification and prioritization of patients based on the severity of their condition using a minimum number of physiological signals. Future actions comprise the validation of system's results with real subjects as well as the packaging of the wearable part of the system.

\section{Acknowledgement}

This work is currently supported by the Greek General Secretariat of Research and Technology [27] and European Union under the project "T1EDK-02489 - IntelTriage"

\section{References}

[1] M. Christien, B. Meester, Naomi, Inter Emerg Nurs, 29, 27-31 (2016)

[2] D.A. Travers, A.E. Waller, J.M. Bowling, D. Flowers, J. Tintinalli, J Emerg Nurs, 28, 395-200 (2002)

[3] E.R. Weinerman, R.S. Ratner, A. Robbins, M.A. Lavenhar, AJPH, 56, 1037-1056 (1966)

[4] G.F. Gerald, G.A. Jelinek, D. Scott, M.F. Gerdtz, J Emerg Med, 27, 86-92 (2010)

[5] G. Hollis, P. Sprivulis, EMA, 8, 231-234 (1996)

[6] G.A. Jelinek, M. Little, EM, 8, 226-230 (2009)

[7] S. Dilley, P. Standen, EMA, 10, 12-18 (1998)

[8] M. van Veen, E.W Steyerberg, M. Ruige, et al, BMJ, 337, 7 (2008)

[9] R. Beveridge, JEM, 16, 507-511 (1998)

[10] M.J. Bullard, B. Unger, J. Spence, E. Grafstein, CJEM, 10, 136-151 (2008)

[11] M. Murray, M. Bullard, E. Grafstein, CJEM, 6, 421-427 (2004)

[12] C.M. Fernandes, P. Tanabe, N. Gilboy, et al, J Emerg Nurs, 31, 39-50 (2005)

[13] K. Goransson, A. Ehrenberg R, M. Ehnfors, AMIA Ann Symp Proc, 851 (2003)

[14] J.G. Jimanez, M.J. Murray, R. Beveridge, et al, CJEM, 5, 315-322 (2003)

[15]I. Wulp, M. Baar, A. Schrijuers, JEM, 25, 431-434 (2008)

[16] M. Kyranou, G. Geogiadis, A. Tsiuiki, J Emerg Nurs, 31, 428 (2005)

[17] The MGH/MF Waveform Database: https://physionet.org/physiobank/database/mghdb/ 
[18] A. Adeli, M. Neshat, Proc of the IMECS, 1 (2010)

[19] R.K.C. Billones, M.P. Vicmudo, E.P. Dadios, Inter Con on HNICEM (2015)

[20]F. Dernoncourt, "Introduction to fuzzy logic", MIT (2013)

[21] "Fuzzy Control Programming", International Electrotechnical Commission (IEC), document 61131-7 (1997)

[22] M. Patel, S.K.L. Lal, D. Kavanagh, P. Rossiter, J Expert Systems with Applications, 38, 7235-7242 (2011)

[23] O. Ondracek, J. Pucik, E. Cocherova, Conference: Trends in Biomedical Engineering, University of Zilina (2005).

[24] J.J. Bailey, A.S. Berson, A. Garson, L.G. Horan, et al, JACC, 49, 1109-1127 (2007)

[25] C.R. Lambert, G.A. Imperi, C.J. Pepine, AJC, 58, 225-229 (1986)

[26] Normal Vital Signs - Medscape Reference:

https://emedicine.medscape.com/article/2172054-overview

[27] General Secretariat for Research and Technology: www.gsrt.gr 